\title{
QUEEN'S
UNIVERSITY
BELFAST
}

\section{Robust positive invariance and ultimate boundedness of nonlinear systems}

Bitsoris, G., Vassilaki, M., \& Athanasopoulos, N. (2012). Robust positive invariance and ultimate boundedness of nonlinear systems. In Proceedings of the 20th Mediterranean Conference on Control \& Automation Institute of Electrical and Electronics Engineers Inc.. https://doi.org/10.1109/MED.2012.6265703

\section{Published in:}

Proceedings of the 20th Mediterranean Conference on Control \& Automation

\section{Document Version:}

Peer reviewed version

Queen's University Belfast - Research Portal:

Link to publication record in Queen's University Belfast Research Portal

\section{General rights}

Copyright for the publications made accessible via the Queen's University Belfast Research Portal is retained by the author(s) and / or other copyright owners and it is a condition of accessing these publications that users recognise and abide by the legal requirements associated with these rights.

Take down policy

The Research Portal is Queen's institutional repository that provides access to Queen's research output. Every effort has been made to ensure that content in the Research Portal does not infringe any person's rights, or applicable UK laws. If you discover content in the Research Portal that you believe breaches copyright or violates any law, please contact openaccess@qub.ac.uk. 


\title{
Robust Positive Invariance and Ultimate Boundedness of Nonlinear Systems
}

\author{
George Bitsoris, Marina Vassilaki, and Nikolaos Athanasopoulos
}

\begin{abstract}
In this article the problem of characterizing sets, described by vector nonlinear inequalities of the form $v(x) \leq w$, as robustly positively invariant and targets of uniformly ultimate bounded nonlinear systems is investigated. The class of general parameter uncertain continuous-time dynamical systems affected by exogenous disturbances is considered. The approach is based on establishing an associated monotone nonlinear comparison system. A numerical example is presented to illustrate the approach.
\end{abstract}

\section{INTRODUCTION}

The dynamics of most real world systems involve nonlinearities in the state space description as well as timevarying terms. These terms represent either time varying or imprecisely known parameters and/or persistent external disturbances. Model uncertainties can also been expressed as persistent external disturbances. Two distinct approaches are used for studying this class of systems. The first one consists in considering the uncertain parameters as random variables with known statistics and the external disturbances as stochastic signals. In the second approach only the bounds of variations of parameter uncertainties and external disturbances are known. In this paper the second approach is adopted.

A significant amount of research has been done on the positive invariance of polyhedral sets of linear continuoustime systems, e.g. [1]-[6] and [7] including the references therein. A considerable work has also been devoted to special classes of nonlinear systems not only for the analysis but also for the design problem [8]-[15].

The objective of this paper is the establishment of conditions guaranteeing the positive invariance and/or ultimate boundedness in subsets of system's state space described by nonlinear vector inequalities of the form $v(x) \leq w$. This class of sets is general enough to include convex and nonconvex sets, possibly unbounded, which can be non-connected. The idea behind the proposed approach lies in the association of the original system with a quasi-monotone comparison system. It is shown that existence of a special structured robust invariant set for the comparison system is a necessary and sufficient condition for positive invariance of the corresponding set in the original system space. Moreover, the problem of robust uniform ultimate boundedness of nonlinear

G. Bitsoris is with the Control Systems Laboratory of the Electrical and Computer Engineering Department, University of Patras, 26500 Patras, Greece. bitsoris@ece. upatras.gr

M. Vassilaki is with the Department of Electrical Engineering, School of Pedagogical and Technological Education, Heraklion 141 21, Athens, Greece. mvassilaki@aspete.gr

N. Athanasopoulos is with the Electrical Engineering Department, Eindhoven University of Technology, 5600 MB, Eindhoven, The Netherlands. n. athanasopoulosetue.nl continuous-time systems with uncertain parameters and/or exogenous disturbances is considered. In this case also, a comparison system scheme is used. Conditions for a set to be a domain of attraction of the target set of robust uniform ultimate bounded systems are also given.

The paper is organized as follows: In the following section, the basic notation and definitions are given. In section III, necessary and sufficient conditions guaranteeing robust positive invariance for general sets described by nonlinear vector inequalities are developed. In section IV, the uniform ultimate boundedness of nonlinear systems is investigated. Finaly, a numerical example illustrating the proposed approach is given in section $\mathrm{V}$.

\section{Preliminaries}

Throughout the paper, capital letters denote real matrices and lower case letters denote column vectors or scalars. $\mathbb{R}^{n}$ denotes the real $n$-space and $\mathbb{R}^{n \times m}$ denotes the set of real $n \times m$ matrices. For two $n \times m$ real matrices $A=\left(a_{i j}\right)$, and $B=\left(b_{i j}\right)$, the inequality $A \leq B(A<B)$ is equivalent to $a_{i j} \leq b_{i j}\left(a_{i j}<b_{i j}\right)$. Similar notation holds for vectors. Finally, $\mathscr{T}$ denotes the time set $\mathscr{T}=[0, \infty)$ and $I_{q}$ denotes the $q \times q$ identity matrix.

We consider time-varying uncertain nonlinear systems with disturbances described by a differential equation of the form

$$
\dot{x}(t)=f(t, x(t), \zeta, \eta(t))
$$

where $x \in \mathbb{R}^{n}$ is the system state , $t \in \mathscr{T}$ is the time variable, $\zeta$ represents uncertain parameters and $\eta(t)$ represents unknown external disturbances or model uncertainties. Uncertain parameters $\zeta$ are assumed to belong to a subset $\mathscr{Z}$ of $\mathbb{R}^{s_{\zeta}}$. Functions $\eta(t)$ are uknown but belong to the set $\Omega_{\eta}$ of bounded piecewise continuous functions $\eta: \mathscr{T} \rightarrow$ $\mathscr{H}$ where $\mathscr{H}$ is assumed to be a compact subset of $\mathbb{R}^{s_{\eta}}$ containing the origin as an interior point. It is also assumed that $f: \mathscr{T} \times \mathbb{R}^{n} \times \mathscr{Z} \times \mathscr{H} \rightarrow \mathbb{R}^{n}$ is a continuous function satisfying sufficient conditions guaranteeing the existence of a unique solution $x\left(t ; t_{0}, x_{0}\right)$ for every initial condition $x_{0}\left(t_{0}\right)=x_{0} \in \mathbb{R}^{n}, t_{0} \in \mathscr{T}, t \geq t_{0}, \zeta \in \mathscr{Z}$ and any function $\eta(t) \in \Omega_{\eta}$.

An important subclass of systems (1) are nonlinear systems with both parameter uncertainties and additive input disturbances $\eta(t)$ described by differential equations of the form

$$
\dot{x}(t)=g(x(t), \zeta)+E \eta(t)
$$

where $g: \mathbb{R}^{n} \times \mathscr{Z} \rightarrow \mathbb{R}^{n}$ and $E \in \mathbb{R}^{n \times s_{\eta}}$. It is assumed that $g(x, \zeta)$ is a continuous function satisfying sufficient 
conditions guaranteeing the existence of a unique solution $x\left(t ; t_{0}, x_{0}\right)$ of system (2) for every initial condition $x_{0}\left(t_{0}\right)=$ $x_{0} \in \mathbb{R}^{n}, t \geq t_{0}, \zeta \in \mathscr{Z}$ and any function $\eta(\cdot) \in \Omega_{\eta}$.

The quasi-monotone nondecreasing functions defined below play an important role in the development of the results of this paper:

Definition 1. A vector valued function $h(t, y, \zeta, \eta), h$ : $\mathscr{T} \times \mathbb{R}^{q} \times \mathscr{Z} \times \mathscr{H} \rightarrow \mathbb{R}^{q}$ is said to be quasi-monotone nondecreasing if for any $\zeta \in \mathscr{Z}, \eta(t) \in \Omega_{\eta}$ and $t \in \mathscr{T}$ all its components $h_{i}\left(t, y_{1}, y_{2}, \ldots y_{q}, \zeta, \eta\right) \quad i=1,2, \ldots, q$ are nondecreasing with respect to $y_{j} j=1,2, \ldots, q, \quad j \neq i$.

If function $h(t, y, \zeta, \eta)$ is quasi-monotone nondecreasing, then for any $\zeta \in \mathscr{Z}$ and $\eta \in \mathscr{H}$ system

$$
\dot{y}(t)=h(t, y(t), \zeta, \eta(t))
$$

is monotone, in the sense that $y_{0} \leq \hat{y}_{0}$ implies $y\left(t ; t_{0}, y_{0}\right) \leq$ $y\left(t ; t_{0}, \hat{y}_{0}\right)$ for all $\zeta \in \mathscr{Z}, \eta(\cdot) \in \Omega_{\eta}, t_{0} \in \mathscr{T}$ and $t \geq t_{0}$. If, in addition, $h(t, 0, \zeta, \eta) \equiv 0$ then [16] $y\left(t ; t_{0}, y_{0}\right) \geq 0$ for all $y_{0} \in \mathbb{R}^{q}, t_{0} \in \mathscr{T}$ and $t \geq t_{0}$.

Given a continuous function $v(x), v: \mathbb{R}^{n} \rightarrow \mathbb{R}^{q}, \dot{v}_{(1)}(x)$ denotes its total time derivative w.r.t. to system (1) and is defined by relation

$$
\dot{v}_{(1)}(x(t))=\lim _{\Delta t \rightarrow 0^{+}} \sup \frac{v(x(t+\Delta t))-v(x(t))}{\Delta t} .
$$

In the sequel, it is assumed that the total time derivative of function $v(x)$ w.r.t. system (1) exists for all $x \in \mathbb{R}^{n}$, $t \in \mathscr{T}, \zeta \in \mathscr{Z}$ and any disturbance $\eta(t) \in \Omega_{\eta}$. In the case of a differentiable function $v(x)$ the total time derivative of function $v(x)$ w.r.t. system (1) is computed by relation

$$
\dot{v}_{(1)}(x)=\frac{\partial v(x)}{\partial x} f(t, x, \zeta, \eta(t))
$$

Consider now a vector valued function $v(x), \quad v$ : $\mathbb{R}^{n} \rightarrow \mathbb{R}^{q}$ and a quasi-monotone nondecreasing function $h(t, y, \zeta, \eta), \quad h: \mathscr{T} \times \mathbb{R}^{q} \times \mathscr{Z} \times \mathscr{H} \rightarrow \mathbb{R}^{q}$ satisfying inequality

$$
\dot{v}_{(1)}(x) \leq h_{i}(t, v(x), \zeta, \eta(t)) \quad i=1,2, \ldots, q .
$$

Then the system

$$
\dot{y}=h(t, y, \zeta, \eta(t))
$$

is said to be a comparison system of system (1) associated with the vector valued function $v(x)$. If function $h(t, y, \zeta, \eta)$ satisfies sufficient conditions guaranteeing the existence of a unique and continuous solution $y\left(t ; t_{0}, y_{0}\right)$ of the comparison system (7) for all $y_{0} \in \mathbb{R}^{q}, t_{0} \in \mathscr{T}, \zeta \in \mathscr{Z}$ and any disturbance $\eta(t) \in \Omega_{\eta}$ then for system (1) and its comparison system (7), the following result holds:

Lemma 1. If (7) is a comparison system of system (1) associated with the vector valued function $v(x)$, then for any $y_{0} \in \mathbb{R}^{q}$ the inequality $v\left(x_{0}\right) \leq y_{0}$ implies $v\left(x\left(t ; t_{0}, x_{0}\right)\right) \leq$ $y\left(t ; t_{0}, y_{0}\right)$ for all $t \geq t_{0}$.

In the sequel, it is assumed that functions $v(x)$ are continuous and satisfy sufficient conditions for the existence of a unique continuous solution $y\left(t ; t_{0}, y_{0}\right)$ of the comparison system (7) for all $y_{0} \in \mathbb{R}^{q}, t_{0} \in \mathscr{T}, \zeta \in \mathscr{Z}$ and any disturbance $\eta(t) \in \Omega_{\eta}$.

\section{POSITIVE INVARIANCE}

We consider uncertain systems described by state equations (1).

Definition 2: A subset $\Delta$ of the state space of system (1) is said to be robustly positively invariant (RPI) if for any $t_{0} \in \mathscr{T}, \zeta \in \mathscr{Z}, \eta(t) \in \Omega_{\eta}$ and every initial condition $x\left(t_{0}\right)=x_{0} \in \Delta$ the corresponding trajectory remains in $\Delta$, that is $x\left(t ; t_{0}, x_{0}\right) \in \Delta$ for all $t \geq t_{0}$.

In this section we are interested in the positive invariance of sets $P(v, w) \subset \mathbb{R}^{n}$ defined by a relation of the form

$$
P(v, w) \stackrel{\text { def }}{=}\left\{x \in \mathbb{R}^{n}: v(x) \leq w\right\}
$$

where $w \in \mathbb{R}^{q}$ and $v(x), v: \mathbb{R}^{n} \longrightarrow \mathbb{R}^{q}$ is a continuous vector valued function such that its total time derivative $\dot{v}_{(1)}(x)$ exists for any $\zeta \in \mathscr{Z}$ and any disturbance $\eta(t) \in \Omega_{\eta}$. Functions $v(x)$ are assumed to be of class $R$, according to the following definition:

Definition 3. The vector valued function $v(x)=$ $\left[\begin{array}{ll}v_{1}(x) & v_{2}(x) \ldots v_{s}(x)\end{array}\right]^{T}, \quad v: \mathbb{R}^{n} \rightarrow \mathbb{R}^{q}, v(0)=0$ is said to be of class $R$ in a set $\mathscr{Y} \subset \mathbb{R}^{q}$ if, given a $y \in \mathscr{Y}$, for any index $i$ with $1 \leq i \leq q$ there exists a $x \in \mathbb{R}^{n}$ such that $v_{i}(x)=y_{i}$ and $v_{j}(x) \leq y_{j}$ for $j \neq i$.

It is clear that if $v(x)$ is a function of class $R$ in $\mathscr{Y}$, then for any $y \in \mathscr{Y}$ the corresponding sets

$P_{(i)}(v, y) \stackrel{\text { def }}{=}\left\{x \in \mathbb{R}^{n}: v_{i}(x)=y_{i}, v_{j}(x) \leq y_{j} j=1,2, \ldots, q j \neq i\right\}$

are non empty.

It is also assumed that the associated sets

$$
S_{(i)}(v, f, y) \stackrel{\text { def }}{=}\left\{z \in \mathbb{R}:\left(\exists x \in P_{(i)}(v, y): z=\dot{v}_{i(1)}(x)\right)\right\}
$$

for $i=1,2, \ldots, q$ are compact for all $y \in \mathscr{Y}$.

In the following theorem necessary and sufficient conditions for set $P(v, w)$ to be positively invariant with respect to system (1) are established.

Theorem 1. Given a function $v(x), v: \mathbb{R}^{n} \longrightarrow \mathbb{R}^{q}$ of class $R$ in $\mathscr{Y}$ and a vector $w \in \mathscr{Y}$, the set $P(v, w)$ is positively invariant with respect to system (1) if and only if there exists a quasi-monotone nondecreasing function $h(t, y, \zeta, \eta), h$ : $\mathscr{T} \times \mathscr{Y} \times \mathscr{Z} \times \mathscr{H} \rightarrow \mathbb{R}^{q}$ such that

$$
\dot{v}_{(1)}(x(t)) \leq h(t, v(x), \zeta, \eta(t))
$$

and

$$
h(t, w, \zeta, \eta(t)) \leq 0 \quad \forall t \in \mathscr{T}, \zeta \in \mathscr{Z}, \forall \eta(t) \in \Omega_{\eta} .
$$

Proof. a) Sufficiency: Since function $h(t, y, \zeta, \eta)$ has been assumed to satisfy conditions guaranteeing the existence of a unique continuous solution $y\left(t ; t_{0}, y_{0}\right)$ of system (7) for all $y_{0} \in \mathbb{R}^{q}, t_{0} \in \mathscr{T}, \zeta \in \mathscr{Z}$ and any disturbance $\eta(t) \in \Omega_{\eta}$, from (12) and the hypothesis that function $h(t, y, \zeta, \eta(t))$ is quasimonotone nondecreasing, it follows that $y\left(t ; t_{0}, y_{0}\right) \leq w$ for all $t \geq t_{0}$ [16]. Since, in addition functions $h(t, y, \zeta, \eta)$ and $v(x)$ satisfy (11), system (7) is a comparison system of system 
(1) associated with the vector valued function $v(x)$. Thus, by virtue of Lemma $1, v\left(x_{0}\right) \leq y_{0}$ implies $v\left[x\left(t ; t_{0}, x_{0}\right)\right] \leq$ $y\left(t ; t_{0}, y_{0}\right)$ for all $t \geq t_{0}, \zeta \in \mathscr{Z}$ and any $\eta(t) \in \Omega_{\eta}$. Thus, if $v\left(x_{0}\right) \leq w$ then $v\left[x\left(t ; t_{0}, x_{0}\right)\right] \leq w$ for all $t \geq t_{0}$. Consequently, $P(v, w)$ is positively invariant with respect to system (1).

b) Necessity: Consider the functions $h_{i}: \mathscr{T} \times \mathscr{Y} \times$ $\mathscr{Z} \times \mathscr{H} \rightarrow \mathbb{R}, \quad$ defined by the relations

$$
h_{i}(t, y, \zeta, \eta)=\max _{x(t) \in P_{(i)}(v, y)}\left\{\dot{v}_{(1) i}(x(t))\right\} \quad i=1,2, \ldots, q .
$$

with $P_{(i)}(v, y)$ given by (9). Since function $v(x)$ is of class $R$ in $\mathscr{Y}$, sets $P_{(i)}(v, y) i=1,2, \ldots, q$ are non empty for all $y \in \mathscr{Y}$.Since, in addition, sets $S\left(v_{i}, f, y\right)$ defined by (10) have assumed be compact, the function $h(t, y, \zeta, \eta(t))=$ $\left[h_{1}(t, y, \zeta, \eta(t)) \quad h_{2}(t, y, \zeta, \eta(t)) \ldots h_{q}(t, y, \zeta, \eta(t))\right]^{T} \quad$ exists and, by definition, is quasi-monotone nondecreasing. Furthermore, $\dot{v}(x)_{(1)} \leq h(t, v(x), \zeta, \eta(t))$ for any $x \in \mathbb{R}^{n}$ such that $v(x)=y, y \in \mathscr{Y}$. Therefore, the system $\dot{y}(t)=h(t, y(t), \zeta, \eta(t))$ is a comparison system of system (1) associated with the vector valued function $v(x)$. Condition (12) is also satisfied because, otherwise, there would exist an index $i$ such that $h_{i}(t, w, \zeta, \eta(t))>0$. Then, by definition of the function $h(t, y, \zeta, \eta(t))$, there would also exist a $t_{0}$ and a state $x_{0}$ such that $v_{i}\left(x_{0}\right)=w_{i}$ and $v_{j}\left(x_{0}\right) \leq w_{j}$ for $j=1,2, \ldots, q j \neq i$, such that $\dot{v}_{i}\left(x_{0}\right)_{(1)}=h_{i}\left(t_{0}, w, \zeta, \eta(t)\right)>0$. This, however, would imply that the component $v_{i}\left(x\left(t_{0} ; t_{0}, x_{0}\right)\right)=v_{i}\left(x_{0}\right)=w_{i}$ of the function $v\left(x\left(t ; t_{0} x_{0}\right)\right)$ would be increasing on $t=t_{0}$ along the trajectory $x\left(t ; t_{0}, x_{0}\right)$, thus contradicting the hypothesis that $P(v, w)$ is positively invariant. Therefore, (12) is also satisfied.

Remark 1. It is clear from the proof of the above theorem, that it is not necessary the comparison system to be defined in the whole space $\mathbb{R}^{q}$. The positive invariance of the set $P(v, w)$ is guaranteed if, for example, the hypotheses of the Theorem 1 are satisfied for a comparison system defined in set $\mathscr{Y}$. Furthermore, from the first part of the proof (sufficiency), it follows that in order to verify that a set $P(v, w)$ is positively invariant it is sufficient to examine whether conditions (11) and (12) are verified for some quasimonotone nondecreasing function $h(t, y, \zeta, \eta(t))$ defined in a subset of $\mathscr{Y}$ such that $w \in \mathscr{Y}$.

Remark 2. It is clear that function $h(t, y, \zeta, \eta(t))$ is not unique. However, it can be proven that if $y\left(t ; t_{0}, v\left(x_{0}\right)\right)$ denotes the trajectories of the comparison system defined by (13), then the corresponding trajectory $y^{*}\left(t ; t_{0}, v\left(x_{0}\right)\right)$ of any other comparison system

$$
\dot{y}^{*}(t)=h^{*}\left(t, y^{*}(t), \zeta, \eta(t)\right)
$$

defined by a relation

$$
\dot{v}_{(1)}(x(t)) \leq h^{*}(t, v(x(t)), \zeta, \eta(t))
$$

satisfies the inequality

$$
v\left(x\left(t ; t_{0}, x_{0}\right)\right) \leq y\left(t ; t_{0}, v\left(x_{0}\right)\right) \leq y^{*}\left(t ; t_{0}, v\left(x_{0}\right)\right) \quad \forall t \geq t_{0}
$$

where $v_{0}=v\left(x_{0}\right)$. Therefore the trajectories $y\left(t ; t_{0}, v_{0}\right)$ of a comparison system defined by (13) are the least upper bounds of $v\left(x\left(t ; t_{0}, x_{0}\right)\right)$. For this reason, a comparison system defined by (13) is said to be optimal.

Next, we use this result for establishing conditions of positive invariance of a polyhedral set

$$
R(G, w) \stackrel{\text { def }}{=}\left\{x \in \mathbb{R}^{n}: G x \leq w\right\}
$$

$G \in \mathbb{R}^{q \times n}, w \in \mathbb{R}^{q}$ with respect to the important class of nonlinear systems with both parameter uncertainties $\zeta$ and additive input disturbances $\eta(t)$ described by differential equations (2).

Theorem 2. The polyhedral set $R(G, w)$ is robustly positively invariant set w.r.t system (2) if and only if there exists a quasi-monotone nondecreasing function $h^{*}(y, \zeta), h^{*}$ : $\mathscr{Y} \times \mathscr{Z} \rightarrow \mathbb{R}^{q}$ such that

$$
\begin{gathered}
G g(x, \zeta) \leq h^{*}(G x, \zeta) \\
h^{*}(w, \zeta)+d \leq 0 \quad \forall \zeta \in \mathscr{Z} \quad \forall t \in \mathscr{T}
\end{gathered}
$$

where $d=\left[\begin{array}{llll}d_{1} & d_{2} & \cdots & d_{q}\end{array}\right]^{\mathscr{T}}$,

$$
d_{i}=\max _{\eta \in \mathscr{H}}\left\{(G E \eta)_{i}\right\} \quad i=1,2, \ldots, q
$$

Proof. Since $R(G, w)=P(v, w)$ with $v(x)=G x$, according to Theorem.1, set $R(G, w)$ is positively invariant w.r.t. system (2) if and only if there exists a quasi-monotone nondecreasing function $h(t, v(x), \zeta, \eta(t))$ satisfying conditions (11) and (12). For system (2), these conditions are written as

$$
\dot{v}_{(1)}(x(t))=G g(x(t), \zeta)+G E \eta(t) \leq h(t, v(x), \zeta, \eta(t))
$$

and

$$
h(t, w, \zeta, \eta(t)) \leq 0 \quad \forall t \in \mathscr{T}, \zeta \in \mathscr{Z}, \forall \eta(t) \in \Omega_{\eta}
$$

respectively. Condition (17) is satisfied by setting

$$
h(t, y, \zeta, \eta(t))=h^{*}(y, \zeta)+d
$$

with $h^{*}(y, \zeta)$ and $d$ satisfying conditions (14) and (16) respectively. Then, from (15) it follows that condition (18) is also satisfied.

\section{ULTIMATE BOUNDEDNESS OF NONLINEAR SYSTEMS}

The presence of unknown exogenous disturbances may exclude the existence of an equilibrium state for system (1). This is certainly the case when these disturbances are additive. In such case one is interested in the ultimate boundedness of the system according to the following defnition.

Let $\mathscr{X}$ be a compact subset of the state space $\mathbb{R}^{n}$ containing the origin as an interior point.

Definition 3. System (1) is said to be robustly uniformly ultimately bounded (RUUB) in a subset $\mathscr{X}$ of the state space $\mathbb{R}^{n}$ if there exists a set $\mathscr{D}, \mathscr{X} \subset \mathscr{D} \subseteq \mathbb{R}^{n}$ such that for any $\zeta \in \mathscr{Z}$ and $\eta(\cdot) \in \Omega_{\eta}$ and every initial condition $x\left(t_{0}\right)=x_{0} \in$ $\mathscr{D}$ there exists an instant $t^{*}\left(x_{0}\right)$ such that $x\left(t ; t_{0}, x_{0}\right) \in \mathscr{X}$ for all $t \geq t_{0}+t^{*}\left(x_{0}\right)$. Set $\mathscr{D}$ is said to be a domain of attraction of the uniformly ultimately bounded set $\mathscr{X}$. 
It is clear that if system (1) is robustly uniformly ultimately bounded in a positively invariant set $\mathscr{X}$, then $\mathscr{D}$ is a domain of attraction if and only if for each initial state $x_{0} \in \mathscr{D}$ there exists a $t^{*}\left(x_{0}\right)$ such that $x\left(t ; t_{0}, x_{0}\right) \in \mathscr{X}$ for $t=t^{*}\left(x_{0}\right)$. Let $d(x, \mathscr{X})$ denote the distance of state $x$ from set $\mathscr{X}$. Then, we can give the following definition:

Definition 4. A subset $\mathscr{X}$ of the state space of system (1) is said to be robustly uniformly asymptotically stable if, for any $\zeta \in \mathscr{Z}$ and $\eta(\cdot) \in \Omega_{\eta}$,

a) given a $t_{0} \in \mathscr{T}$ and a $\varepsilon>0$ there exists $\delta(\varepsilon)>0$ such that $d\left(x_{0}, \mathscr{X}\right)<\delta(\varepsilon)$ implies $d\left(x\left(t ; t_{0}, x_{0}\right), \mathscr{X}\right)<\varepsilon$ for all $t_{0} \in \mathscr{T}$ and $t \geq t_{0}$

b) there exists a set $\mathscr{D}, \mathscr{X} \subset \mathscr{D} \subseteq \mathbb{R}^{n}$ such that $\lim _{t \rightarrow \infty} d\left(x\left(t ; t_{0}, x_{0}\right), \mathscr{X}\right)=0$ for any $t_{0} \in \mathscr{T}$ and every initial condition $x\left(t_{0}\right)=x_{0} \in \mathscr{D}$.

Set $\mathscr{D}$ is said to be a domain of attraction of the robustly uniformly asymptotically stable s set $\mathscr{X}$.

In the following Lemma, we establish conditions of robust uniform ultimate boundedness of monotone systems

$$
\dot{y}=h(t, y, \zeta, \eta(t))
$$

with $h(t, y, \zeta, \eta))$ being a quasi-monotone nondecreasing function:

Lemma 2. If there exist positive real numbers $r_{1}, r_{2}$, and $\varepsilon$ such that

$$
h(t, r w, \zeta, \eta) \leq-\varepsilon r w \quad \forall r \in\left[r_{1}, r_{2}\right], \quad \forall t \in \mathscr{T}
$$

for all $\zeta \in \mathscr{Z}$ and $\eta(\cdot) \in \Omega_{\eta}$, then system (20) is robustly uniformly ultimately bounded in set $R\left(I_{q}, r_{1} w\right)$ with $R\left(I_{q}, r_{2} w\right)$ as domain of attraction.

Proof. Consider the linear system

$$
\dot{z}(t)=-\varepsilon z(t)
$$

where $z \in \mathbb{R}^{q}$. Then,

$$
z\left(t ; t_{0}, r_{2} w\right)=r_{2} w e^{-\varepsilon\left(t-t_{0}\right)} \quad \forall t \geq t_{0}
$$

and

$$
z\left(t ; t_{0}, r_{2} w\right) \leq r_{1} w \quad \forall t \geq t_{0}+t^{*}
$$

with $t^{*}$ given by

$$
t^{*}=\frac{1}{\varepsilon} \log \frac{r_{2}}{r_{1}}
$$

We claim that, for any $\zeta \in \mathscr{Z}$ and $\eta(\cdot) \in \Omega_{\eta}$,

$$
y\left(t ; t_{0}, r_{2} w\right) \leq z\left(t ; t_{0}, r_{2} w\right) \quad \forall t \in\left[t_{0}, t_{0}+t^{*}\right]
$$

It is clear that inequality (25) is satisfied for $t=t_{0}$. This is also true for all $t \in\left[t_{0}, t_{0}+t^{*}\right]$ because otherwise there would exist a time instant $t \in\left[t_{0}, t_{0}+t^{*}\right]$ and an index $j$ such that

$$
\begin{gathered}
y_{i}\left(t ; t_{0}, r_{2} w\right) \leq z_{i}\left(t ; t_{0}, r_{2} w\right) \quad i=1,2, \ldots, q \quad i \neq j \\
y_{j}\left(t ; t_{0}, r_{2} w\right)=z_{j}\left(t ; t_{0}, r_{2} w\right)
\end{gathered}
$$

and $y_{j}\left(t+\delta t ; t_{0}, r_{2} w\right)>z_{j}\left(t+\delta t ; t_{0}, r_{2} w\right)$ for all $\delta t$ belonging to a time-interval $(0, \Delta t)$. The latter relation, however, could not be verified because, taking into account that function $h(t, y, \zeta, \eta)$ is quasi-monotone nondecreasing, from (26), (27), (23), and (24) it would follow that

$$
\begin{aligned}
& \dot{y}_{j}\left(t ; t_{0}, r_{2} w\right)-\dot{z}_{j}\left(t ; t_{0}, r_{2} w\right)= \\
= & h_{j}\left(t, y\left(t ; t_{0}, r_{2} w\right), \zeta, \eta\right)+\varepsilon z_{j}\left(t ; t_{0}, r_{2} w\right) \\
\leq & h_{j}\left(t, z\left(t ; t_{0}, r_{2} w\right), \zeta, \eta\right)+\varepsilon z_{j}\left(t ; t_{0}, r_{2} w\right) \\
\leq & h_{j}\left(t, e^{-\varepsilon\left(t-t_{0}\right)} r_{2} w, \zeta, \eta\right)+\varepsilon e^{-\varepsilon\left(t-t_{0}\right)} r_{2} w \\
\leq & -\varepsilon e^{-\varepsilon\left(t-t_{0}\right)} r_{2} w+\varepsilon e^{-\varepsilon\left(t-t_{0}\right)} r_{2} w=0
\end{aligned}
$$

for all $t \geq t_{0}$ such that

$$
r_{1} \leq e^{-\varepsilon\left(t-t_{0}\right)} r_{2} \leq r_{2}
$$

or equivalently, such that

$$
t_{0} \leq t \leq t_{0}+t^{*}
$$

Therefore, (25) is indeed satisfied.

Now, let $y_{0} \in R\left(1_{q}, r_{2} w\right)$. Then $y_{0} \leq r_{2} w$ and since system (20) is monotone, it follows that

$$
y\left(t ; t_{0,} y_{0}\right) \leq y\left(t ; t_{0}, r_{2} w\right),
$$

or all $t_{0} \in \mathscr{T}$ and $t \geq t_{0}$. This inequality together with (26) and (24) implies that

$$
y\left(t ; t_{0, y_{0}}\right) \leq r_{1} w \quad \forall t \geq t_{0}+t^{*} .
$$

This, in turn,implies that $R\left(I_{q}, r_{1} w\right)$ is a robustly uniformly ultimately bounded set of system (20) and $R\left(I_{q}, r_{1} w\right)$ is a domain of attraction.

Let us consider subsets $\mathscr{X}$ of system's state space that include the set of all possible equilibrium states of the system, that is, $\mathscr{X}_{0} \subseteq \mathscr{X}$ where

$\mathscr{X}_{0}=\left\{x \in \mathbb{R}^{n}:(\exists \zeta \in \mathscr{Z}, \eta \in \mathscr{H}: f(t, x, \zeta, \eta)=0 \forall t \in \mathscr{T})\right\}$

In the following theorem, necessary and sufficient conditions for the robust uniform ultimate boundedness of systems (1) in set $\mathscr{X}$ are established:

Theorem 3. If for a continuous function $v(x), v: \mathbb{R}^{n} \rightarrow$ $\mathbb{R}^{q}$ there exist a quasi-monotone nondecreasing function $h(t, y, \zeta, \eta), h: \mathscr{T} \times \mathscr{Y} \times \mathscr{Z} \times \mathscr{H} \rightarrow \mathbb{R}^{q}$ and positive real numbers $r_{1}, r_{2}$, and $\varepsilon$ such that

$$
\begin{gathered}
P\left(v, r_{1} w\right) \subseteq \mathscr{X} \subset P\left(v, r_{2} w\right) \\
\dot{v}_{(1)}(x(t)) \leq h(t, v(x), \zeta, \eta) \\
h(t, r w, \zeta, \eta) \leq-r \varepsilon w \quad \forall r \in\left[r_{1}, r_{2}\right], \quad \forall t \in \mathscr{T}
\end{gathered}
$$

then system (1) is robustly uniformly ultimately bounded in set $\mathscr{X}$ and $P\left(v, r_{2} w\right)$ is a domain of attraction.

Proof. According to Lemma 2, from condition (31) it follows that $R\left(I_{q}, r_{1} w\right)$ is a robustly uniformly ultimately bounded set of system (20) and $R\left(I_{q}, r_{2} w\right)$ is as domain of attraction. Since, function $h(t, y, \zeta, \eta)$ is quasi-monotone 
nondecreasing, from (30) it follows that system is a comparison system of (20) associated with the vector valued function $v(x)$. Therefore,

$$
v\left(x_{0}\right) \leq y_{0}
$$

implies

$$
v\left(x\left(t ; t_{0}, x_{0}\right)\right) \leq y\left(t ; t_{0}, y_{0}\right) \quad \forall t \geq t_{0} .
$$

Now, if $x_{0} \in P\left(v, r_{2} w\right)$ then, by virtue of (29),

$$
v\left(x_{0}\right) \leq r_{2} w
$$

and setting

$$
y_{0}=r_{2} w
$$

from (32)-(33) it follows that

$$
v\left(x\left(t ; t_{0}, x_{0}\right)\right) \leq y\left(t ; t_{0}, r_{2} w\right) \quad \forall t \geq t_{0} .
$$

This implies that there exists a $t^{*}$ such that

$$
v\left(x\left(t ; t_{0}, x_{0}\right)\right) \leq r_{1} w \quad \forall t \geq t_{0}+t^{*}
$$

because, $R\left(I_{q}, r_{1} w\right)$ is a robustly uniformly ultimately bounded set of system by conditions (20) and $R\left(I_{q}, r_{2} w\right)$ a corresponding domain of attraction. Consequently, if $x_{0} \in$ $P\left(v, r_{2} w\right)$ then $v\left(x\left(t ; t_{0}, x_{0}\right)\right) \leq r_{1} w \quad \forall t \geq t_{0}+t^{*}$ or equivalently $v\left(x\left(t ; t_{0}, x_{0}\right)\right) \in P\left(v, r_{1} w\right)$, that is by virtue of (29) $v\left(x\left(t ; t_{0}, x_{0}\right)\right) \in \mathscr{X}, \forall t \geq t_{0}+t^{*}$. Therefore, $\mathscr{X}$ is a robustly uniformly ultimately bounded set $\mathscr{X}$ of system (1) and $P\left(v, r_{2} w\right)$ is a domain of attraction.

Remark 3. From the proof of Lemma 2, it follows that, under the hypotheses of this theorem, all initial states $x_{0}$ belonging to the domain of attraction $P\left(v, r_{2} w\right)$ are transferred to the target set $\mathscr{X}$ in a time non exceeding $t_{\min }$ where

$$
t_{\min }=\frac{1}{\varepsilon} \log \frac{r_{2}}{r_{1}} \text {. }
$$

Remark 4. In section III, it has been shown that the existence of a quasi-monototone nondecreasing function $h(t, y, \zeta, \eta(t))$ satisfying inequality (30) and inequality $h(t, w, \zeta, \eta) \leq 0$ for all $\zeta \in \mathscr{Z}, \eta(\cdot) \in \Omega_{\eta}$ and $\forall t \in \mathscr{T}$, is a necessary and sufficient condition for the positive invariance of set $P(v, w)$ w.r.t. system (1). Therefore, under the hypotheses of Theorem 3 , besides robust uniform ultimate stability in set $\mathscr{X}$, the positive invariance of all sets $P(v, r w)$ $\forall r \in\left[r_{1}, r_{2}\right]$ is guaranteed.

Next, we use this result for establishing conditions of uniform ultimate stability of system in a polyhedral set

$$
R(G, w) \stackrel{\text { def }}{=}\left\{x \in \mathbb{R}^{n}: G x \leq w\right\}
$$

$G \in \mathbb{R}^{q \times n}, w \in \mathbb{R}^{q}$ with respect to the important class of nonlinear systems with both parameter uncertainties $\zeta$ and input additive disturbances $\eta(t)$ described by differential equations of the form

$$
\dot{x}(t)=g(t, x(t), \zeta)+E \eta(t)
$$

A direct consequence of Theorem 3 is the following result:
Theorem 4. If there exists a quasi-monotone nondecreasing function $h^{*}(y, \zeta), h^{*}: \mathscr{Y} \times \mathscr{Z} \rightarrow \mathbb{R}^{q}$ such that

$$
\begin{gathered}
P\left(v, r_{1} w\right) \subseteq \mathscr{X} \subset P\left(v, r_{2} w\right) \\
G g(x, \zeta) \leq h^{*}(G x, \zeta) \\
h^{*}(r w, \zeta)+d \leq-r \varepsilon w \quad \forall r \in\left[r_{1}, r_{2}\right], \quad \forall \zeta \in \mathscr{Z},
\end{gathered}
$$

where $d=\left[\begin{array}{llll}d_{1} & d_{2} & \cdots & d_{q 1}\end{array}\right]^{\mathscr{T}}$,

$$
d_{i}=\max _{\eta \in \Omega_{\eta}}\left\{(G E \eta)_{i}\right\} \quad i=1,2, \ldots, q
$$

then $\mathscr{X}$ is ultimately bounded and $P\left(v, r_{2} w\right)$ is a domain of attraction of system (37).

\section{NUMERICAL EXAMPLE}

In order to illustrate the results established in Sections III and IV, we provide a numerical example of a control problem for bilinear dynamical systems.

Let us consider the first order bilinear dynamical system

$$
\dot{x}(t)=-1.15 x(t)+u(t)+0.1 x(t) u(t)+\eta(t)
$$

where $\eta(t) \in \Omega_{\eta}, \Omega_{\eta}$ being the set of piecewise continuous functions from $\mathscr{T}$ to $\mathscr{H}=\left[\begin{array}{ll}-0.3 & 1\end{array}\right]$. The problem concerns the determination of a linear state-feedback control law

$$
u(t)=l x(t)
$$

such that the resulting closed-loop system

$$
\dot{x}(t)=(-1.15+l) x(t)+0.1 l x^{2}(t)+\eta(t)
$$

is robustly uniformly ultimately bounded in the region

$$
\mathscr{X}=\{x \in \mathbb{R} \mid-0.5 \leq x \leq 0.7\},
$$

and

$$
\mathscr{D}=\{x \in \mathbb{R} \mid-1 \leq x \leq 1.4\}
$$

is a corresponding domain of attraction.

Setting $v(x)=G x$ with

$$
G=\left[\begin{array}{c}
1 \\
-1
\end{array}\right], \quad w=\left[\begin{array}{l}
0.7 \\
0.5
\end{array}\right]
$$

sets $\mathscr{X}$ and $\mathscr{D}$ can be equivalently written as $\mathscr{X}=R(G, w)=$ $\{x \in \mathbb{R}: G x \leq w\}$ and $\mathscr{D}=R(G, 2 w)$. Therefore, according to Theorem 4, the control law (39) is a solution to the control problem if there exists a quasi-monotone nondecreasing function $h^{*}(y)$ and a positive real number $\varepsilon$ such that

$$
G g(x)=\left[\begin{array}{c}
(-1.15+l) x(t)+0.1 l x^{2}(t) \\
-(-1.15+l) x(t)-0.1 l x^{2}(t)
\end{array}\right] \leq h^{*}(G x)
$$

and

$$
h^{*}(r w)+d \leq-\varepsilon r w \quad \forall r \in[1,2],
$$

where

$$
d=\left[\begin{array}{|}
\max _{\eta \in[-0.3,1]}\{\eta\} \\
\max _{\eta \in[-0.3,1]}\{-\eta\}
\end{array}\right]=\left[\begin{array}{c}
1 \\
0.3
\end{array}\right]
$$


Condition (43) is satisfied for

$$
h^{*}(y)=\left[\begin{array}{c}
(-1.15+l) y_{1}+0.1 \max \{0, l\} \max \left\{y_{1}^{2}, y_{2}^{2}\right\} \\
(-1.15+l) y_{2}+0.1 \max \{0,-l\} \max \left\{y_{1}^{2}, y_{2}^{2}\right\}
\end{array}\right]
$$

It is clear that function $h^{*}(y)$ is quasi monotone nondecreasing for $y_{1} \geq 0, y_{2} \geq 0$. Thus condition (44) becomes

$$
h^{*}(r w)+d \leq-\varepsilon r\left[\begin{array}{l}
0.7 \\
0.5
\end{array}\right] \quad \forall r \in[1,2],
$$

where

$$
h^{*}(r w)=\left[\begin{array}{c}
(-1.15+l) 0.7 r+0.1 \max \{0, l\}(0.49) r^{2}+ \\
(-1.15+l) 0.5 r+0.1 \max \{0,-l\}(0.49) r^{2}
\end{array}\right] .
$$

It is a simple task to show that this inequality is satisfied if

$$
l \leq-0.279
$$

\section{CONCLUSIONS}

In this article the robust positive invariance of sets described by nonlinear inequalities of the form $v(x) \leq w$ and the uniform ultimate boundedness of nonlinear systems has been investigated. The class of general parameter uncertain continuous-time dynamical systems affected by exogenous disturbances is considered. The approach presented here is based on the establishment of a monotone nonlinear comparison system and then deriving positive invariance and uniform ultimate boundedness properties for the original system from the corresponding properties of the comparison system. It is worth noting that the so obtained conditions of positive invariance are both necessary and sufficient.

\section{REFERENCES}

[1] G. Bitsoris. Existence of positively invariant polyhedral sets for continuous-time linear systems, Control Theory Adv. Technol. vol. , vol, 7, pp. 407-427, 1991.

[2] E.B. Castelan and J.C. Hennet, "Invariant polyhedra of continuoustime linear systems", IEEE Transactions on Automatic Control, Vol. 38, 1993, pp. $1680-85$.

[3] C.E.T. Dorea and J.C. Hennet, "On robust positive invariance of unbounded polyhedra", Proceedings of the IFAC Conference on System Structure and Control, pp.243-248, 1995.

[4] J. W. Nieuwenhuis, About Positive Invariance and Asymptotic Stability. Appl Math Optim pp. 407-427, 1991.

[5] B. E. A. Milani,Ultimate Boundedness Sets for Continuous-time Linear Systems with Deadzone Feedback Controls, in Proceedings of the 44th IEEE Conference on Decision and Control, and the European Control Conference, pp. 6853-6858, Seville, Spain, 2005.

[6] A. Barabanov, R. Ortega, G. Escobar,On ultimate boundedness around non-assignable equilibria of linear time-invariant systems, Automatica, vol. 44 , pp. $286-288,2008$

[7] F. Blanchini, and S. Miani. Set-theoretic methods in control. Systems and Control: Foundations and Applications, Birkhauser, 2008.

[8] A.T. Ademola, P.O. Arawomo, On the Stability and Ultimate Boundedness of Solutions for Certain Third Order Differential Equations, Journal of Mathematics and Statistics, vol. 4 pp. 201-207, 2008.

[9] B. Brogliato, A. Trofino Neto, Practical Stabilization of a Class of Nonlinear Systems with Partially Known Uncertainties, Automatica, vol. 31 pp. 145-150, 1995.

[10] B. R. Barmish,I. R. Petersen, A. Feuer, Linear Ultimate Boundedness Control of Uncertain Dynamical Systems, Automatica, vol. 19, pp. 523532, 1983.

[11] Fenlin, C. Yanrong, L. Junyong, Adaptive Robust Output Feedback Controllers Guaranteeing Uniform Ultimate Boundedness for Uncertain Nonlinear Systems, in Proceeding of the 2004 American Control Conference, pp. 3693 - 36982004.
[12] G. Bitsoris, M. Vassilaki and E. Gravalou Comparison principle and constrained regulation of continuous-time systems, in Proceedings of the 3rd European Control Conference, 1995.

[13] G. Makay, Uniform Boundedness and Uniform Ultimate Boundedness for Functional Differential Equations, Funkcialaj Ekvaciojvol. 38, pp. 283-296, 1995.

[14] J. Peuteman, D. Aeyels, R. Sepulchre, Boundedness Properties for the Time-Varying Nonlinear Systems, SIAM J. Control Optim. vol. 39, $1408-1422,2000$.

[15] A.P. Tchangani, M. Dambrine, J.P. Richard, Stability, attraction domains, and ultimate boundedness for nonlinear neutral systems, Mathematics and Computers in Simulation, vol. 45, pp. 291-298, 1998.

[16] G. Bitsoris, Stability analysis of nonlinear dynamical systems. International Journal of Control, vol. 38, pp. 699 - 711, 1983. 